\title{
Interactive comment on "Role of black carbon in the formation of primary organic aerosols: Insights from molecular dynamics simulations" by Xiaoqi Zhou et al.
}

Xiaoxiang Wang (Referee)

xiaoxiang.wang@mpic.de

Received and published: 16 March 2020

Dear Authors,

Yes, you can address these issues after the epidemic.

Please contact the editorial office if you need an extension of "review and discussion".

Best regards, Xiaoxiang 